\title{
Dynamische Methode zur Berechnung thermodynamischer Gleichgewichte in reaktiven Mehrphasensystemen
}

\begin{tabular}{|r|l|}
\hline Journal: & Chemie Ingenieur Technik \\
\hline Manuscript ID & cite.201600070.R2 \\
\hline Diley - Manuscript type: & Forschungsarbeit \\
\hline Complete List of Authors: & $\begin{array}{l}\text { Zinser, Alexander; Otto-von-Guericke Universität, Lehrstuhl } \\
\text { Systemverfahrenstechnik } \\
\text { Sundmacher, Kai; Otto-von-Guericke Universität, Lehrstuhl } \\
\text { Systemverfahrenstechnik; Max-Planck-Institut für Dynamik komplexer } \\
\text { technischer Systeme, Prozesstechnik }\end{array}$ \\
\hline Keywords: & Phasengleichgewichte, Reaktionsgleichgewichte, Mehrphasensysteme \\
\hline & \\
\hline
\end{tabular}

SCHOLARONE ${ }^{\text {' }}$

Manuscripts 


\title{
Dynamische Methode zur Berechnung thermodynamischer Gleichgewichte in reaktiven Mehrphasensystemen*
}

\author{
A. Zinser ${ }^{1}$, K. Sundmacher ${ }^{1,2}$ \\ ${ }^{1}$ Otto-von-Guericke Universität, Lehrstuhl Systemverfahrenstechnik, Universitätsplatz 2, 39106 Magdeburg \\ ${ }^{2}$ Max-Planck-Institut für Dynamik komplexer technischer Systeme, Sandtorstraße 1, 39106 Magdeburg \\ ${ }^{*}$ Herrn Prof. Dr.-Ing. Andreas Seidel-Morgenstern zum 60. Geburtstag gewidmet
}

\begin{abstract}
Ein allgemeiner Ansatz zur Berechnung thermodynamischer Gleichgewichte für nichtreaktive und reaktive Mehrphasensysteme wird vorgestellt. Dieser basiert auf der Simulation der dynamischen Evolution eines Gemisches aus einem beliebigen Nichtgleichgewichtszustand in das thermodynamische Gleichgewicht hinein. Der Vorteil dieses Ansatzes ist, dass er komplexe Mehrkomponentensysteme behandeln kann, ohne numerische Schwierigkeiten zu bereiten. Die Anwendung der vorgestellten Methode wird anhand verschiedener Gleichgewichtssituationen des Stoffsystems der Butylacetat-Synthese erläutert.
\end{abstract}

\section{Einleitung}

Bei der Simulation und Optimierung von verfahrenstechnischen Prozessen spielt die Berechnung von thermodynamischen Gleichgewichten eine zentrale Rolle. So müssen Reaktionsgleichgewichte in chemischen Reaktoren ebenso berechnet werden, wie Phasengleichgewichte in unterschiedlichen Trennprozessen. In verfahrenstechnischen Prozessen treten häufig auch unterschiedliche Typen von Phasengleichgewichten auf, wie zum Beispiel Dampf-Flüssig-Gleichgewichte (VLE) oder Flüssig-Flüssig-Gleichgewichte (LLE).

Zur Berechnung thermodynamischer Gleichgewichte haben sich zwei mathematische Formulierungen als Ausgangspunkt etabliert. In beiden Fällen wird ein geschlossenes System bei konstantem Druck $P$ und konstanter Temperatur $T$ betrachtet.

Die erste Formulierung ist ein Optimierungsproblem: die Minimierung der Gibbs-Energie des Systems unter Berücksichtigung der Atomzahlbilanzen als Gleichungsnebenbedingungen [1] [2] [3]. Dieses Optimierungsproblem wird mit Hilfe von Lagrange-Multiplikatoren in ein unbeschränktes Optimierungsproblem überführt und kann dann durch leistungsfähige Algorithmen für NLP-Probleme, z. B. mit einer Gradientenmethode, gelöst werden [3]. Die numerische Effizienz hängt $u$. a. von den gewählten Startwerten ab.

Die zweite Formulierung ist ein nichtlineares algebraisches Gleichungssystem: das Erfüllen der notwendigen Bedingungen, die am Minimum der Gibbs-Funktion gelten. Dies entspricht der formalen Lösung der Optimalitätsbedingungen der ersten Formulierung. Für ein nichtreaktives System mit $p$ Phasen und $s$ Spezies ist dies die Gleichheit der chemischen Potentiale in allen Phasen [1]:

$$
\mu_{\alpha}^{\pi}=\mu_{\alpha}^{\pi^{\prime}}, \quad \forall \alpha \in \mathcal{S}, \quad \forall \pi \neq \pi^{\prime} \in \mathcal{P}
$$


Dies ist ein nichtlineares algebraisches Gleichungssystem (NAE) mit $s p(p-1) / 2$ Unbekannten. Die Lösung kann z. B. mit Hilfe von Newton-Solvern oder auf Gleichgewichtsberechnungen spezialisierten Algorithmen [4] [5] erfolgen. Um zügige Konvergenz zu erreichen, werden auch hier gute Startwerte benötigt. Wie im Fall der Minimierung der Gibbs-Energie kann es Konvergenzprobleme bis hin zum Abbruch der Lösungssuche kommen.

Neben den beiden hier erwähnten Methoden sind in der Literatur weitere Verfahren zur Berechnung thermodynamischer Gleichgewichte bekannt, wie z. B. die Minimierung der Gibbs-Energie unter Verwendung globaler Optimierungsalgorithmen [6] oder der Optimierung auf Basis des Dualitätsprinzips [7]. Eine Übersicht weiterer globaler Verfahren einschließlich eines Vergleiches ihrer numerischen Effizienz ist in [8] dargestellt.

Wünschenswert wäre es also, über eine Berechnungsmethode zu verfügen, die auch bei stark nichtidealem Stoffverhalten für ein- und mehrphasige Vielstoffsysteme den Gleichgewichtszustand für beliebige Startwerte stets zuverlässig und in angemessener Rechengeschwindigkeit liefert. Eine solche Methode ist die dynamische Simulation eines geschlossenen Nichtgleichgewichtssystems [9] [10] [11]. Sie basiert auf der numerischen Lösung gewöhnlicher Differentialgleichungen. Die Lösung algebraischer Gleichungssysteme mittels Relaxation eines Differentialgleichungssystems geht in der Verfahrenstechnik zurück auf Ketchum (1979) [12].

Diese dynamische Methode soll im Folgenden kurz allgemein entwickelt und dann für ein konkretes Beispiel, das Gemisch der Reaktanten der Butylacetat-Synthese, angewendet werden. Ein Matlab-Code wird durch die Autoren gern zur Verfügung gestellt.

\section{Formulierung der dynamischen Methode}

Wir definieren die Menge aller Phasen $\mathcal{P}$, die im betrachteten System auftreten können. Im Beispiel eines Dampf-Flüssig-Flüssig-Systems (VLL) mit einer Gasphase (V) und zwei koexistierenden Flüssigphasen (L1 und L2) ist diese $\mathcal{P}=\{\mathrm{V}, \mathrm{L} 1, \mathrm{~L} 2\}$. Zusätzlich definieren wir die Menge aller im System auftretenden Spezies $\mathcal{S}$.

Zur Berechnung eines Phasengleichgewichtes zwischen $p$ Phasen in einem System mit $s$ Komponenten werden bis zu $s p(p-1) / 2$ Ratengleichungen $r_{\alpha}^{\pi, \pi^{\prime}}$ benötigt. Diese Ratengleichungen beschreiben die molaren Stoffströme für alle Komponenten $\alpha \in \mathcal{S}$ zwischen allen Kombinationen von Phasen $\pi$ und $\pi^{\prime}$ mit $\pi, \pi^{\prime} \in \mathcal{P}$ und $\pi \neq \pi^{\prime}$. Weiterhin kann in jeder Phase $\pi \in \mathcal{P}$ ein Satz chemischer Reaktionen $\rho \in \mathcal{R}^{\pi}$ existieren.

Der vorgeschlagene Ansatz zur Berechnung des thermodynamischen Gleichgewichtes eines reaktiven Mehrphasensystems basiert nun auf der Lösung des Differentialgleichungssystems

$$
\frac{\mathrm{d} \mathbf{n}}{\mathrm{d} \tau}=\mathbf{A r}
$$

welches die dynamische Entwicklung der Stoffmengen in allen Phasen

$$
\mathbf{n}=\left[\mathbf{n}^{\pi}\right]_{\pi \in \mathcal{P}}, \quad \text { mit } \quad \mathbf{n}^{\pi}=\left[n_{\alpha}^{\pi}\right]_{\alpha \in \mathcal{S}}
$$


über die Zeit $\tau$ beschreibt. Dabei bezeichnet $\mathbf{A}$ eine verallgemeinerte stöchiometrische Matrix und $\mathbf{r}$ den Vektor der Ratengleichungen, der sowohl den Stoffaustausch zwischen den einzelnen Phasen beschreibt also auch die Quellen und Senken der einzelnen Komponenten infolge chemischer Reaktionen. Die stöchiometrische Matrix

$$
\mathbf{A}=\left[\begin{array}{ll}
\mathbf{A}_{\mathrm{p}} & \mathbf{A}_{\mathrm{r}}
\end{array}\right]
$$

wird partitioniert in eine Teilmatrix $\mathbf{A}_{\mathrm{p}}$, welche die Interaktionen zwischen den Phasen modelliert und eine Teilmatrix $\mathbf{A}_{\mathbf{r}}$, die die Stöchiometrie der chemischen Reaktionen in jeder Phase beschreibt. Analog zerlegen wir den Vektor der Ratengleichungen

$$
\mathbf{r}=\left[\begin{array}{l}
\mathbf{r}_{\mathrm{p}} \\
\mathbf{r}_{\mathrm{r}}
\end{array}\right]
$$

in zwei Teile, nämlich den Teilvektor $\mathbf{r}_{\mathrm{p}}$ für die Stoffströme infolge von Phasenübergängen

$$
\mathbf{r}_{\mathrm{p}}=\left[\mathbf{r}^{\pi, \pi^{\prime}}\right]_{\substack{\pi, \pi^{\prime} \in \mathcal{P} \\ \pi \neq \pi^{\prime}}} \text { mit } \mathbf{r}^{\pi, \pi^{\prime}}=\left[r_{\alpha}^{\pi, \pi^{\prime}}\right]_{\alpha \in \mathcal{S}^{\prime}}
$$

und einen Teilvektor $\mathbf{r}_{\mathbf{r}}$ für die Quellen und Senken infolge chemischer Reaktionen:

$$
\mathbf{r}_{\mathrm{r}}=\left[\mathbf{r}^{\pi}\right]_{\pi \in \mathcal{P}} \text { mit } \mathbf{r}^{\pi}=\left[r_{\rho}^{\pi}\right]_{\rho \in \mathcal{R}^{\pi}}
$$

Im nächsten Schritt werden alle Ratengleichungen thermodynamisch konsistent formuliert. Dies führt dazu, dass im stationären Zustand des resultierenden Systems gewöhnlicher Differentialgleichungen die notwendigen Bedingungen des thermodynamischen Gleichgewichtszustands erfüllt sind.

Wir betrachten ein geschlossenes System in einem Thermostaten, so dass $T=$ const. Weiterhin sei durch einen Kolben $P=$ const eingestellt. Das gesamte System möge sich bereits auf diese $T, P$-Niveaus eingeschwungen haben, so dass überall isotherm-isobare Bedingungen vorliegen. Dann gilt für die Gibbs-Energie des Systems, siehe z. B. [13],

$$
\frac{\mathrm{d} G}{\mathrm{~d} \tau}=-T \dot{\sigma_{\mathrm{S}}} \leq 0
$$

Die Entropieproduktionsrate $\dot{\sigma}_{\mathrm{S}}$ ist eine stets nicht-negative Größe, weshalb bei der Evolution des Systems von einem beliebigen Startpunkt hin zum Gleichgewichtszustand die GibbsEnergie stets kontinuierlich abnehmen muss. Diese Aussage gilt unabhängig von der Zahl der Komponenten und Phasen sowohl in nichtreaktiven wie auch in reaktiven Gemischen.

Wir betrachten nun ein solches Nichtgleichgewichtssystem mit $s$ verschiedenen chemischen Spezies. Darin mögen insgesamt $p$ verschiedene Phasen existieren, nämlich eine Gas- bzw. Dampfphase und $(p-1)$ flüssige Phasen. Feste Phasen sollen - ohne Einschränkung der Allgemeingültigkeit der nachfolgenden Herleitung - aus Gründen der Vereinfachung ausgeschlossen werden. Weiterhin sollen in den Phasen auch chemische Reaktionen zwischen den Spezies ablaufen können.

Der Zustand jeder einzelnen Phase wird durch $(s+2)$ Zustandsgrößen beschrieben. Da $T$ und $P$ bekannt sind, verbleiben $s$ Zustandsgrößen, also für das gesamte System $(s p)$ Zustandsgrößen. Wir wählen hierfür die Stoffmengen $n_{\alpha}^{\pi}$ mit $\alpha \in \mathcal{S}$ und $\pi \in \mathcal{P}$. Da sich das System in einem Nichtgleichgewichtszustand befindet, existieren $s(p-1)$ thermo- 
dynamische Triebkräfte für den Stofftransport zwischen zwei beliebigen Phasen $\pi \neq \pi^{\prime} \in \mathcal{P}$, sowie je eine thermodynamische Triebkraft für jede chemische Reaktion im System. Im Einklang mit den Ergebnissen der irreversiblen Thermodynamik, siehe z. B. [14], schreiben wir in Anlehnung an einen Vorschlag von De Donder, siehe z. B. [15], folgende Ratengleichungen für den Stofftransport bzw. für die chemischen Reaktionen an

$$
\begin{aligned}
r_{\alpha}^{\pi, \pi^{\prime}} & =\tilde{k}_{\alpha}^{\pi, \pi^{\prime}}\left(1-e^{-\frac{\Delta_{\mathrm{m}} g_{\alpha}^{\pi, \pi^{\prime}}}{R T}}\right), \text { mit } \Delta_{\mathrm{m}} g_{\alpha}^{\pi, \pi^{\prime}}=\mu_{\alpha}^{\pi}-\mu_{\alpha}^{\pi^{\prime}} \\
r_{\rho}^{\pi} & =\tilde{k}_{\rho}^{\pi}\left(1-e^{-\frac{\Delta_{\mathrm{r}} g_{\rho}^{\pi}}{R T}}\right), \text { mit } \Delta_{\mathrm{r}} g_{\rho}^{\pi}=\sum_{\alpha \in \mathcal{S}} v_{\alpha \rho}^{\pi} \mu_{\alpha}^{\pi}
\end{aligned}
$$

Beide Ratengleichungen sind konsistent mit den notwendigen Bedingungen, die im Phasengleichgewicht bzw. im chemischen Gleichgewicht erfüllt sein müssen. Dann gilt nämlich

$$
\begin{gathered}
\left(\Delta_{\mathrm{m}} g_{\alpha}^{\pi, \pi^{\prime}}\right)_{\mathrm{eq}}=\mu_{\alpha}^{\pi}-\mu_{\alpha}^{\pi^{\prime}}=0 \Rightarrow r_{\alpha, \mathrm{eq}}^{\pi, \pi^{\prime}}=0 \\
\left(\Delta_{\mathrm{r}} g_{\rho}^{\pi}\right)_{\mathrm{eq}}=\sum_{\alpha \in \mathcal{S}} v_{\alpha \rho}^{\pi} \mu_{\alpha}^{\pi}=0 \Rightarrow r_{\rho, \mathrm{eq}}^{\pi}=0
\end{gathered}
$$

Wir stellen nun die Stoffmengenbilanzen für alle Spezies $\alpha \in \mathcal{S}$ in allen Phasen $\pi \in \mathcal{P}$ auf und fassen mit Hilfe der Vektor-Matrix-Schreibweise zusammen:

$$
\frac{\mathrm{d} \mathbf{n}}{\mathrm{d} \tau}=\mathbf{A r}
$$

Dabei werden die Stoffmengenbilanzen über die stöchiometrische Matrix $\mathbf{A}$ mit den jeweiligen Ratengleichungen gekoppelt. Die stöchiometrische Matrix hat für ein zweiphasiges System die Form

$$
\mathbf{A}=\left[\begin{array}{ccc}
-\mathbf{I} & \mathbf{A}_{\mathrm{r}}^{\pi_{1}} & \\
\mathbf{I} & & \mathbf{A}_{\mathrm{r}}^{\pi_{2}}
\end{array}\right]
$$

und für ein dreiphasiges System die Form

$$
\mathbf{A}=\left[\begin{array}{cccccc}
-\mathbf{I} & -\mathbf{I} & & \mathbf{A}_{\mathrm{r}}^{\pi_{1}} & & \\
\mathbf{I} & & -\mathbf{I} & & \mathbf{A}_{\mathrm{r}}^{\pi_{2}} & \\
& \mathbf{I} & \mathbf{I} & & & \mathbf{A}_{\mathrm{r}}^{\pi_{3}}
\end{array}\right] .
$$

Dabei ist I eine Einheitsmatrix der Dimension $s \times s$, der linke Teil der Matrix beschreibt die Interaktionen zwischen den einzelnen Phasen infolge Stofftransportes über eine Phasengrenze hinweg und der rechte Teil modelliert die chemischen Reaktionen in den Phasen über die jeweiligen stöchiometrischen Matrizen $\mathbf{A}_{\mathrm{r}}^{\pi}$. Tritt in einer Phase keine chemische Reaktion auf, so ist die zugehörige stöchiometrische Matrix eine Nullmatrix der Dimension $s \times 0$.

Es liegt nun ein gekoppeltes System nichtlinearer gewöhnlicher Differentialgleichungen (ODE) vor, welches die Evolution von einem beliebigen Nichtgleichgewichtszustand aus in einen stationären Zustand beschreibt. Die Integration des ODE-Systems ist allerdings nur dann möglich, wenn zusätzlich geeignete Zustandsgleichungen vorliegen, mit denen die chemischen Potentiale der Spezies in allen beteiligten Phasen bestimmt werden können. 
Zunächst führen wir die chemischen Potentiale der Spezies auf die Fugazitäten zurück:

$$
\mu_{\alpha}^{\pi}\left(T, P, \mathbf{x}^{\pi}\right)=\mu_{\alpha}^{\mathrm{IG}, \theta}\left(T, P^{\theta}\right)+R T \ln \left(\frac{f_{\alpha}^{\pi}\left(T, P, \mathbf{x}^{\pi}\right)}{P^{\theta}}\right)
$$

wobei als Referenzzustand die reine Komponente als ideales Gas bei der Systemtemperatur $T$ und dem Standarddruck $P^{\theta}=101325 \mathrm{~Pa}$ gewählt wird. Die Molenbrüche $x_{\alpha}^{\pi}$ werden aus den Stoffmengen $n_{\alpha}^{\pi}$ in der Phase $\pi$ berechnet:

$$
x_{\alpha}^{\pi}=\frac{n_{\alpha}^{\pi}}{\sum_{\beta \in \mathcal{S}} n_{\beta}^{\pi}} .
$$

Daraus ergeben sich nach Umformulierung folgende modifizierte Ratengleichungen

$$
\begin{aligned}
& r_{\alpha}^{\pi, \pi^{\prime}}=k_{\alpha}^{\pi, \pi^{\prime}} \times\left[\frac{f_{\alpha}^{\pi}}{P^{\theta}}-\frac{f_{\alpha}^{\pi^{\prime}}}{P^{\theta}}\right] \\
& r_{\rho}^{\pi}=k_{\rho}^{\pi} \times\left[\prod_{\substack{\alpha \in \mathcal{S} \\
v_{\alpha \rho}^{\pi}<0}}\left(\frac{f_{\alpha}^{\pi}}{P^{\theta}}\right)^{-v_{\alpha \rho}^{\pi}}-\frac{1}{K_{\mathrm{eq}, \rho}^{\pi}} \prod_{\substack{\alpha \in \mathcal{S} \\
v_{\alpha \rho}^{\pi}>0}}\left(\frac{f_{\alpha}^{\pi}}{P^{\theta}}\right)^{v_{\alpha \rho}^{\pi}}\right]
\end{aligned}
$$

wobei die chemischen Gleichgewichtskonstanten durch

$$
\begin{aligned}
K_{\mathrm{eq}, \rho}^{\pi} & =\exp \left(-\frac{\Delta_{r} g_{\rho}^{\pi \theta}}{R T}\right) \\
\text { mit } \Delta_{r} g_{\rho}^{\pi \theta} & =\sum_{\alpha \in \mathcal{S}} v_{\alpha \rho}^{\pi} \mu_{\alpha}^{\mathrm{IG}, \theta}\left(T, P^{\theta}\right)
\end{aligned}
$$

definiert sind.

Mittels des Fugazitätskoeffizienten $\varphi_{\alpha}^{\pi}$ und des Aktivitätskoeffizienten $\gamma_{\alpha}^{\pi}$ können die Fugazitäten $f_{\alpha}^{\pi}$ als

$$
f_{\alpha}^{\pi}=x_{\alpha}^{\pi} \varphi_{\alpha}^{\pi} P
$$

bzw.

$$
f_{\alpha}^{\pi}=x_{\alpha}^{\pi} \gamma_{\alpha}^{\pi} f_{\alpha}^{\pi 0}
$$

geschrieben werden. Die Formulierung in den Fugazitätskoeffizienten kann sowohl auf Gasphasen, als auch auf Flüssigphasen angewandt werden. Dabei werden die Fugazitätskoeffizienten mit Hilfe einer Zustandsgleichung, z. B. der Soave-Redlich-Kwong (SRK)Zustandsgleichung [16], ermittelt. Die zweite Formulierung verwendet die Aktivitätskoeffizienten $\gamma_{\alpha}^{\pi}$ und wird üblicherweise auf Flüssigphasen angewandt. Die Aktivitätskoeffizienten können mit Hilfe eines geeigneten $G^{E}$-Modells, wie z. B. NRTL oder UNIQUAC, oder auch mit einer Gruppenbeitragsmethode, z.B. UNIFAC, berechnet werden.

Damit können die bekannten Ansätze, wie sie aus den konventionellen Phasengleichgewichtsberechnungen auf Basis algebraischer Gleichungen bekannt sind, auch im Rahmen der dynamischen Methode angewandt werden: Dies sind der $\varphi$ - $\varphi$-Ansatz, sowie der $\varphi-\gamma$ Ansatz für Dampf-Flüssig-Systeme, und der $\gamma$ - $\gamma$-Ansatz für Flüssig-Flüssig-Systeme. 


\section{Anwendung der dynamischen Methode}

In diesem Abschnitt zeigen wir die Anwendung der oben eingeführten dynamischen Methode auf ein konkretes Beispiel: die Flüssigphase-Synthese von Butylacetat (BuOAc). Diese geschieht durch die Veresterung von Essigsäure (HOAc) mit Butanol $(\mathrm{BuOH})$ gemäß der Reaktionsgleichung [17]

$$
\mathrm{BuOH}+\mathrm{HOAc} \rightleftharpoons \mathrm{BuOAc}+\mathrm{H}_{2} \mathrm{O} .
$$

Als Nebenprodukt dieser Reaktion entsteht Wasser. Bei einer Temperatur von $T=$ $100 \ldots 120^{\circ} \mathrm{C}$ ist die Umsetzung gleichgewichtslimitiert. Die FlüssigphasenGleichgewichtskonstante hat den Wert $K_{e q, 1}=12,57$ [18]. Wird das Wasser aus der reaktiven Phase entfernt, so kann ein höherer Umsatz erreicht werden. Dieses Prinzip wird zum Beispiel im Rahmen der Reaktivdestillation [18] angewandt. Darüber hinaus bilden die beiden binären Systeme Wasser-Butylacetat und Wasser-Butanol jeweils eine Mischungslücke aus. Es kann neben dem Reaktions- und dem Dampf-Flüssig-Gleichgewicht hier also auch noch ein Flüssig-Flüssig-Gleichgewicht auftreten.

Im Folgenden wird nun am Beispiel der Synthese von Butylacetat gezeigt, wie die dynamische Methode auf reaktive Mehrphasensysteme angewendet werden kann. Die resultierenden ODE-Systeme wurden in MATLAB implementiert und mit Hilfe des MATLABSolvers für steife Differentialgleichungen ,ode $15 \mathrm{~s}$ ' numerisch gelöst [19].

\section{Schritt 1: Reaktionsgleichgewicht}

Zunächst soll ausschließlich die chemische Reaktion betrachtet werden. In dem betrachteten System treten vier Komponenten auf

$$
\mathcal{S}=\left\{\mathrm{H}_{2} \mathrm{O}, \mathrm{BuOH}, \mathrm{HOAc}, \mathrm{BuOAc}\right\}
$$

die durch eine Reaktionsgleichung miteinander gekoppelt sind. Die Ratengleichung für diese Reaktion lautet unter Verwendung des $\gamma$-Ansatzes

$$
r_{1}=(x \gamma)_{\mathrm{BuOH}}(x \gamma)_{\mathrm{HOAc}}-\frac{(x \gamma)_{\mathrm{BuOAc}}(x \gamma)_{\mathrm{H} 2 \mathrm{O}}}{K_{\mathrm{eq}, 1}}
$$

wobei der Ratenkoeffizient auf eins normiert werden kann, da wir lediglich am finalen Gleichgewichtszustand interessiert sind, nicht aber an der tatsächlichen Dynamik des Systems. Die Gleichgewichtkonstante $K_{\text {eq, } 1}$ bezieht sich auf die reinen Komponenten bei $T$ und $P$ des Systems in der flüssigen Phase. Da nur die chemische Reaktion betrachtet wird, werden die Indizes, die sich auf die Phase beziehen, weggelassen und die verkürzte Schreibweise $(x \gamma)_{\alpha}=x_{\alpha} \gamma_{\alpha}$ angewandt. Die Aktivitätskoeffizienten werden mit Hilfe des UNIQUAC-Modells berechnet. Die binären Parameter für das UNIQUAC-Modell für das gegebene System sind z. B. in [18] zu finden.

Die stöchiometrische Matrix für dieses System ist durch

$$
\mathbf{A}=\left[\begin{array}{llll}
1 & -1 & -1 & 1
\end{array}\right]^{\mathrm{T}}
$$


gegeben, wobei sich die Reihenfolge der Komponenten hier an der Reihenfolge der Komponenten in der Menge $\mathcal{S}$ orientiert. Daraus ergibt sich das resultierende Differentialgleichungssystem in den vier Zuständen, die die Zusammensetzung des Systems beschreiben:

$$
\frac{\mathrm{d}}{\mathrm{d} \tau}\left[\begin{array}{c}
n_{\mathrm{H} 2 \mathrm{O}} \\
n_{\mathrm{BuOH}} \\
n_{\mathrm{HOAc}} \\
n_{\mathrm{BuOAc}}
\end{array}\right]=\mathbf{A} r_{1}
$$

Dieses System ist vom Druck $P$ unabhängig. Es wurde für eine Temperatur von $T=110^{\circ} \mathrm{C}$ mit einer stöchiometrischen Feedzusammensetzung als Anfangsbedingung gelöst, bis der stationäre Zustand erreicht war. Der Verlauf der Zusammensetzung ist in Abbildung 1 dargestellt. Im stationären Zustand wird ein Umsatz von 74\% erreicht. Die Aktivitätskoeffizienten bewegen sich über die Simulationsdauer hinweg im Bereich $0,98<\gamma_{\alpha}<1,7$ und dürfen somit nicht vernachlässigt werden.

\section{Schritt 2: Dampf-Flüssig-Gleichgewicht}

Im zweiten Schritt wird das Dampf-Flüssig-Gleichgewicht des Reaktionsgemisches betrachtet. Es werden also zwei Phasen, $\mathcal{P}=\{\mathrm{V}, \mathrm{L}\}$, und eine Anfangszusammensetzung des Gesamtsystems von

$$
\mathbf{n}(\tau=0)=\left[\begin{array}{l}
0,74 \mathrm{~mol} \\
0,26 \mathrm{~mol} \\
0,26 \mathrm{~mol} \\
0,74 \mathrm{~mol}
\end{array}\right]
$$

angenommen. Durch das Einführen einer zweiten Phase, der Dampfphase, verdoppelt sich die Anzahl der dynamischen Zustände und es werden Ratengleichungen für den Stofftransport zwischen Dampf- und Flüssigphase benötigt. Mit dem $\varphi$ - $\gamma$-Ansatz folgt die Ratengleichungen

$$
r_{\alpha}^{\mathrm{V}, \mathrm{L}}=x_{\alpha}^{\mathrm{V}} \phi_{\alpha}^{\mathrm{V}} \frac{P}{P^{\circ}}-x_{\alpha}^{\mathrm{L}} \gamma_{\alpha}^{\mathrm{L}} \frac{P_{\text {sat }}(T)}{P^{\circ}}
$$

für die vier Komponenten. Dabei werden die Fugazitätskoeffizienten mit Hilfe der SRK Zustandsgleichung [16] berechnet, die Aktivitätskoeffizienten werden wie im vorherigen Beispiel mit Hilfe UNIQUAC-Modells bestimmt und die Sättigungsdampfdrücke durch eine Antoine-Gleichung [18] bestimmt. Wie im ersten Beispiel können die Ratenkoeffizienten auf eins normiert werden, da wir nur an der korrekten Berechnung des Gleichgewichtszustandes interessiert sind.

Das resultierende ODE-System für den betrachteten Fall, bestehend aus acht dynamischen Zuständen und vier Ratengleichungen, lautet 


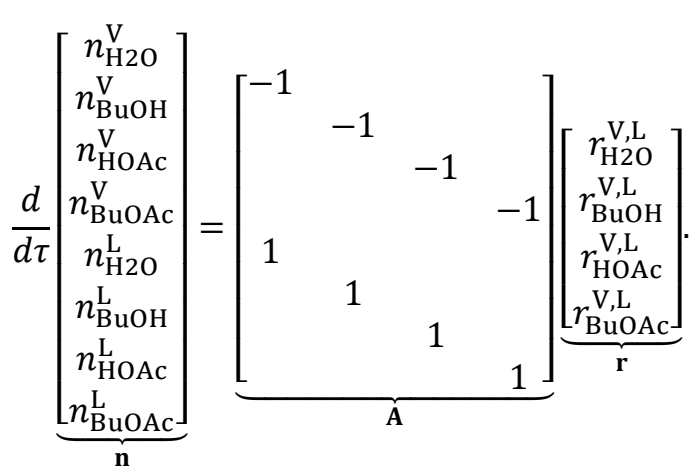

Um dieses ODE-System zu lösen, muss die anfängliche Zusammensetzung des Gesamtsystems noch unter den beiden Phasen aufgeteilt werden. In diesem Fall wurde die Anfangszusammensetzung zu gleichen Teilen unter der Dampf- und Flüssigphase aufgeteilt. Der Verlauf der Zusammensetzungen in beiden Phasen ist in Abbildung 2 dargestellt.

Werden die beiden Edukte, Butanol und Essigsäure, zu einer „Eduktkomponente“ zusammengefasst, so kann die Zusammensetzung einer Phase in einem ternären Phasendiagramm dargestellt werden. Die Trajektorien im ternären Phasendiagramm sind in Abbildung 3, für die reaktive Phase aus Schritt 1, sowie für die beiden Phasen bei der Dampf-Flüssig-Gleichgewichtsberechnung, dargestellt.

\section{Schritt 3: Reaktives Dampf-Flüssig-Gleichgewicht}

Um nun das simultane Reaktions- und Dampf-Flüssig-Gleichgewicht für das betrachtete System zu lösen, könnte man die Schritte 1 und 2 abwechselnd wiederholen, bis sich das simultane Reaktions- und Dampf-Flüssig-Gleichgewicht eingestellt. Diese Vorgehensweise ist jedoch sehr rechenintensiv, da eine Iteration durchgeführt werden muss. Daher wird das reaktive Dampf-Flüssig-Gleichgewicht vorzugsweise simultan berechnet. Zu diesem Zweck werden die Systemgleichungen, die in den beiden Schritten 1 und 2 formuliert wurden, einfach zusammengeführt. Das so erhaltene ODE-System besitzt acht dynamische Zustände und fünf Ratengleichungen, wovon vier auf den Stofftransport über die VL-Phasengrenze und eine auf die chemische Reaktion zurückgehen. Das ODE-System lautet:

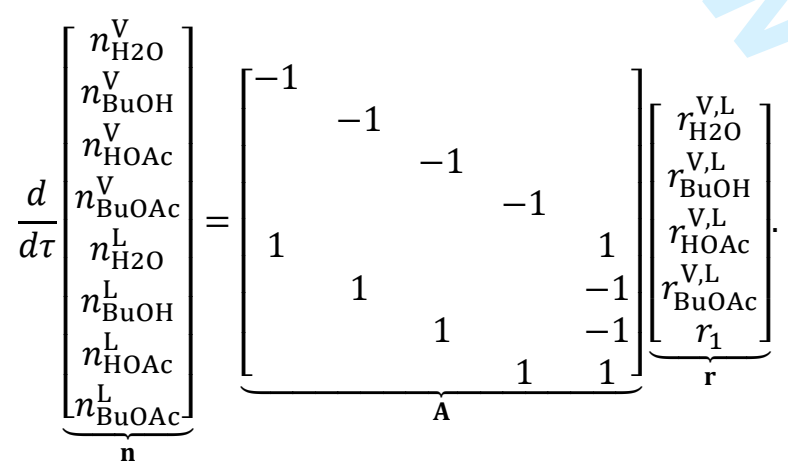

Als Anfangsbedingung wurde ein Reaktionsfortschritt von $\xi=0,5$ angenommen und die resultierende Zusammensetzung zu gleichen Teilen unter der Dampf- und der Flüssigphase aufgeteilt. 
Das ODE-System des reaktiven Dampf-Flüssig-Systems wurde über die Zeit integriert. Der zeitliche Verlauf der Zusammensetzungen in beiden Phasen ist in Abbildung 4 dargestellt. Für die initiale Zusammensetzung stellt sich in der dimensionslosen Zeitskala $\tau=$ $10^{-6} \ldots 10^{-3}$ das Dampf-Flüssig-Gleichgewicht ein, während sich das Reaktionsgleichgewicht im Intervall $\tau=10^{-1} \ldots 10^{2}$ einstellt. Die Trajektorien der beiden Zusammensetzungen im ternären Phasendiagramm sind in Abbildung 5 dargestellt. Es ist gut zu erkennen, dass die Separation im vorliegenden Fall sehr viel schneller abläuft als die Reaktion. Die Zeitkonstanten können aber mit Hilfe der Ratenkoeffizienten für Reaktion und Stofftransport, $k_{\rho}^{\pi}$ bzw. $k_{\alpha}^{\pi, \pi^{\prime}}$, stets so angepasst werden, dass die Zeitkonstanten der beteiligten kinetischen Teilprozesse in etwa gleicher Größenordnung liegen. Für chemische Reaktionen kann diese Normierung z. B. durch $k_{\rho}^{\pi}=\sqrt{K_{\mathrm{eq}, \rho}^{\pi}}$ erfolgen [10].

\section{Schritt 4: Flüssig-Flüssig-Gleichgewicht}

In den eben diskutierten Schritten wurde das System vom einfachen Reaktionsgleichgewicht zum reaktiven Dampf-Flüssig-Gleichgewicht ausgebaut. Da das gewählte Beispielsystem auch in der Lage ist zwei koexistierende Flüssigphasen auszubilden, soll nun das FlüssigFlüssig-Gleichgewicht untersucht werden.

Es werden zwei Flüssigphasen $\mathcal{P}=\{\mathrm{L} 1, \mathrm{~L} 2\}$ betrachtet und der Stofftransport zwischen diesen Phasen wird mit Hilfe des $\gamma$ - $\gamma$-Ansatzes modelliert:

$$
r_{\alpha}^{L 1, L 2}=\left((x \gamma)_{\alpha}^{\mathrm{L} 1}-(x \gamma)_{\alpha}^{\mathrm{L} 2}\right)\left(\frac{f_{\alpha}^{0}}{P^{\theta}}\right),
$$

wobei die Aktivitätskoeffizienten mit Hilfe des UNIQUAC-Modells berechnet werden. Die Fugazitäten der reinen Komponenten $f_{\alpha}^{0}$ sind in den beiden flüssigen Phasen gleich. Der Term $\left(\frac{f_{\alpha}^{0}}{P^{\theta}}\right)$ spielt für die Lage des Flüssig-Flüssig-Gleichgewichts keine Rolle, er kann mit Ratenkoeffizienten zusammengefasst werden. Das resultierende Differentialgleichungssystem hat dieselbe Form wie dasjenige in dem Dampf-FlüssigBeispiel.

Das Flüssig-Flüssig-Gleichgewicht wurde für verschiedene Zusammensetzungen bei $T=100^{\circ} \mathrm{C}$ berechnet, um die Gleichgewichtskurven für die wässrige Phase (L1) und die organische Phase (L2) zu konstruieren. Diese sind in Abbildung 6 in einem ternären Phasendiagramm dargestellt. Dazu wurde die Gesamtzusammensetzung mittels der Reaktionslaufzahl $\xi$ der Butylacetat-Synthese im Intervall $0,7<\xi<1$ variiert. Hier ist zu erkennen, dass ab einer Laufzahl von etwa $\xi=0,7$ genug Wasser gebildet wird, um eine zweite flüssige Phase zu bilden.

\section{Schritt 5: Reaktives Flüssig-Flüssig-Gleichgewicht}

Im letzten Schritt wurde das Flüssig-Flüssig-System aus Schritt 4 um die chemische Reaktion erweitert. Diese kann hier in beiden Flüssigphasen stattfinden. Als Anfangsbedingung wurde eine Reaktionslaufzahl von $\xi=0,75$ und eine zufällige Verteilung unter den beiden Flüssigphasen angenommen. Die Entwicklung der Zusammensetzung in 
beiden Phasen über der Zeit ist in Abbildung 7 dargestellt. Es ist zu erkennen, dass die Gleichgewichtszusammensetzung in beiden Phasen identisch ist. Dies bedeutet, dass das reaktive System nur eine flüssige Phase ausbildet, was sich auch bei Variation der Anfangsbedingungen und der Temperatur nicht änderte.

Dies deutet bei Gültigkeit des verwendeten UNIQUAC-Modells darauf hin, dass eine zweite Flüssigphase im reaktiven System nicht auftritt und in der Simulation vernachlässigt werden kann.

\section{Vergleich der dynamischen Methode mit konventionellen Ansätzen}

In diesem Abschnitt soll die numerische Effizienz der vorgestellten dynamischen Methode bewertet und mit verschiedenen Algorithmen zur Lösung des äquivalenten nichtlinearen algebraischen Gleichungssystems verglichen werden. Dies wird am Beispiel des reaktiven Dampf-Flüssig-Gleichgewichts (Schritt 3) durchgeführt. Das algebraische Gleichungssystem zur Beschreibung dieses Gleichgewichtsproblems besteht aus vier Gleichungen für das Dampf-Flüssig-Gleichgewicht

$$
x_{\alpha}^{\mathrm{V}} \phi_{\alpha}^{\mathrm{V}} \frac{P}{P^{\circ}}=x_{\alpha}^{\mathrm{L}} \gamma_{\alpha}^{\mathrm{L}} \frac{P_{\text {sat }}(T)}{P^{\circ}},
$$

einer Gleichung für das Reaktionsgleichgewicht in der flüssigen Phase

$$
K_{\mathrm{eq}, 1}=\frac{(x \gamma)_{\mathrm{BuOAc}}(x \gamma)_{\mathrm{H} 2 \mathrm{O}}}{(x \gamma)_{\mathrm{BuOH}}(x \gamma)_{\mathrm{HOAc}}},
$$

sowie den drei Atomzahlbilanzen für Kohlenstoff, Wasserstoff und Sauerstoff

$$
\sum_{\alpha \in \mathcal{S}}\left(n_{\alpha}^{\mathrm{V}}+n_{\alpha}^{\mathrm{L}}\right) a_{i \alpha}=n_{\mathrm{atom}, i} \forall i \in\{\mathrm{C}, \mathrm{H}, 0\}
$$

Die Gleichungen für die Atomzahlbilanzen tauchen in der dynamischen Methode nicht explizit auf, sondern sind implizit durch die stöchiometrischen Koeffizienten immer erfüllt.

Das Gleichgewichtsproblem wurde für 20 zufällig gewählten Sätzen von Pseudozufallszahlen als Anfangsbedingung initialisiert und mit Hilfe der Algorithmen

- Trust-Region Dogleg [20],

- Trust-Region Reflective [21] und

- Levenberg-Marquardt [22]

numerisch gelöst und mit der dynamischen Methode verglichen. Die numerische Performance aller vier Verfahren ist in Tabelle 1 zusammengefasst.

Tabelle 1: Vergleich der numerischen Performance der vier verglichenen Verfahren. Die besten Zahlenwerte sind jeweils Fett dargestellt.

\begin{tabular}{|l|c|c|c|c|c|c|c|}
\hline & \multirow{2}{*}{ Konvergenz? } & \multicolumn{2}{|c|}{ Funktionsauswertungen } & \multicolumn{4}{c|}{ CPU-Zeit in ms } \\
\cline { 3 - 8 } & Mittel & Var $^{2}$ & $\min$ & Mittel & $\max$ & Var $^{2}$ \\
\hline $\begin{array}{l}\text { Trust-region } \\
\text { Dogleg }\end{array}$ & $95 \%$ & 193 & 142 & 44 & 134 & 440 & 91 \\
\hline Trust-region & $65 \%$ & 247 & 136 & 70 & 184 & 386 & 109 \\
\hline
\end{tabular}




\begin{tabular}{|l|c|c|c|c|c|c|c|}
\hline Reflective & & & & & & & \\
\hline $\begin{array}{l}\text { Levenberg- } \\
\text { Marquardt }\end{array}$ & $65 \%$ & 218 & 135 & 32 & 109 & 221 & 67 \\
\hline $\begin{array}{l}\text { Dynamische } \\
\text { Methode, } \\
\text { ode15s }\end{array}$ & $\mathbf{1 0 0 \%}$ & 255 & 22 & 87 & 108 & 130 & 10 \\
\hline
\end{tabular}

Dabei ist zunächst festzustellen, dass die vorgestellte Dynamische Methode als einziges Verfahren für alle Sätze an unterschiedlichen Startwerten gegen das thermodynamische Gleichgewicht konvergiert ist, während dies bei der Lösung der algebraischen Gleichungssysteme nur in $65 \%$ bzw. 95\% der getesteten Fälle gelang. Tabelle 1 zeigt auch die durchschnittlich benötigte Anzahl an Funktionsauswerten, sowie deren Standardabweichung, für den Fall der Konvergenz. Dabei ist zu sehen, dass die dynamische Methode zwar im Durchschnitt die meisten Funktionsauswertungen benötigt, dafür aber die geringste Standardabweichung der gemessenen Werte aufweist. In den letzten vier Spalten sind die CPU-Zeiten der Methoden dargestellt. Neben dem Mittelwert und der Standardabweichung sind hier auch die Zeiten des schnellsten und langsamsten Tests dargestellt. Hier ist gut zu erkennen, dass die drei Verfahren zur Lösung der algebraischen Gleichungen in ihrer Effizienz stark von den gewählten Anfangsbedingungen abhängen, während die dynamische Methode in ihrer Effizienz nur wenig von der Anfangskonfiguration des Systems abhängt.

\section{Zusammenfassung}

Neben den beiden gängigen Ansätzen zur Berechnung von thermodynamischen Gleichgewichten, der Minimierung der Gibbs-Energie und der direkten Erfüllung der notwendigen Gleichgewichtsbedingungen, wurde die dynamische Methode als dritter Weg zur Berechnung thermodynamischer Gleichgewichte vorgestellt. Diese Methode basiert auf der Lösung eines Systems gewöhnlicher Differentialgleichungen, welche die Evolution des Systems in den Gleichgewichtszustand beschreiben. Der Ausgangspunkt der vorgestellten dynamischen Methode ist physikalischer Natur: Massenbilanzen und Ratenausdrücke, die aus der Nichtgleichgewichtsthermodynamik abgeleitet sind, bilden das resultierende System gewöhnlicher Differentialgleichungen. Ähnliche Rechenvorschriften könnten durch Anwendungen einer differential evolution-Methode auf das Minimierungsproblem der GibbsEnergie, oder durch Anwendung einer numerical continuation-Methode auf die algebraischen Gleichgewichtsbedingungen, entstehen.

Es ist anzumerken, dass alle drei Ansätze nicht rigoros sind und auch metastabile Zustände als Gleichgewicht liefern können. Ein triviales Beispiel für einen solchen metastabilen Zustand ist ein Flüssig-Flüssig-System in dem beide Phasen die identische Zusammensetzung haben. In diesem Fall ist die notwendige Optimalitätsbedingung erfüllt, bzw. im Falle der vorgestellten dynamischen Methode sind die Ratengleichungen gleich Null. Um einen solchen metastabilen Zustand ausschließen zu können muss neben der notwendigen Bedingung auch die hinreichende Optimalitätsbedingung überprüft werden: die positive Definitheit der Gibbs-Funktion. In der Praxis kann dieses Problem häufig durch Variation der Anfangsbedingungen gelöst werden. 
Grundsätzlich ist die Methode nicht in der Lage, die Anzahl der koexistierenden Phasen vorherzusagen. Aus diesem Grund empfiehlt es sich zunächst eine hinreichende Menge an flüssigen Phasen anzunehmen. Sollten weniger flüssige Phasen koexistieren als zunächst angenommen, so werden im stationären Zustand mehrere Phasen eine identische Zusammensetzung aufweisen und können zusammengefasst werden. Problematischer erweist sich das Verschwinden einer Dampf-Phase, da bei der Lösung der Differentialgleichungen formal auch die Zustandsgleichung der nicht existenten Dampfphase ausgewertet werden muss, was nicht immer gelingt. Hier muss vor der Simulation bereits entschieden werden, ob eine Dampf-Phase existiert.

Die dynamische Methode wurde in weiteren Arbeiten der Autoren erfolgreich für weitere komplexe Reaktions- oder Phasengleichgewichtsprobleme angewendet [9] [10]. Auch wurde die Methode bereits für die Simulation von kontinuierlichen verfahrenstechnischen Prozessen eingesetzt [11]. Eine Erweiterung der dynamischen Methode auf die Optimierung verfahrenstechnischer Prozesse könnte mit zusätzlichen numerischen Kosten verbunden sein: Falls hier gradientenbasierte Verfahren eingesetzt werden, müssen zusätzlich die Sensitivitäten des dynamischen Problems berücksichtigt werden.

Bei der Herleitung der dynamischen Methode wurde in dieser Arbeit von der Nichtgleichgewichtsthermodynamik ausgegangen, während die zitierten Arbeiten der Autoren die Isofugazitätsbedingung als Startpunkt nutzen. Auch behandeln die zitierten Arbeiten in erster Linie nur chemische oder Phasengleichgewichte, während in der vorliegenden Arbeit der Fokus auf der simultanen Berechnung von chemischem und Phasengleichgewicht liegt. Ein Vergleich der dynamischen Methode mit der Minimierung der Gibbs-Energie wurde am Beispiel einer chemischen Gleichgewichtsberechnung in [10] durchgeführt. Ein Vergleich der dynamischen Methode mit der Lösung der algebraischen Gleichgewichtsbedingungen ist der vorliegenden Arbeit zu entnehmen.

Der Ansatz ist durch die Verwendung von Ratenansätzen für Stofftransport und Reaktionen physikalisch motiviert. Er liefert in angemessener Rechenzeit die Zusammensetzungen aller Phasen im Gleichgewichtszustand und sollte insbesondere bei der Gleichgewichtsberechnung reaktiver Mehrphasensysteme als leistungsfähige Alternative zu den etablierten Berechnungsmethoden in Betracht gezogen werden.

\section{Danksagung}

Diese Arbeit entstand im Rahmen des Sonderforschungsbereichs SFB-TRR 63 „Integrierte chemische Prozesse in flüssigen Mehrphasensystemen“ (InPROMPT). Wir danken der Deutschen Forschungsgemeinschaft (DFG) für die Förderung.

\section{Abkürzungen}

\begin{tabular}{|c|c|}
\hline $\mathrm{BuOAc}$ & Butylacetat $\left(\mathrm{CH}_{3}-\mathrm{COO}-\mathrm{CH}_{2}-\mathrm{CH}_{2}-\mathrm{CH}_{2}-\mathrm{CH}_{3}\right)$ \\
\hline $\mathrm{BuOH}$ & Butanol $\left(\mathrm{CH}_{3}-\mathrm{CH}_{2}-\mathrm{CH}_{2}-\mathrm{CH}_{2}-\mathrm{OH}\right)$ \\
\hline HOAC & Essigsäure $\left(\mathrm{CH}_{3}-\mathrm{COOH}\right)$ \\
\hline NRTL & Aktivitätskoeffizientenmodell (№n-ㅁandom Iwo Liquid model) \\
\hline
\end{tabular}




\begin{tabular}{|c|c|}
\hline ODE & gewöhnliche Differentialgleichung (ôrdinary differential equation) \\
\hline UNIFAC & $\begin{array}{l}\text { Aktivitätskoeffizientenmodell (Universal Quasichemical Functional Group } \\
\text { Activity Coefficients) }\end{array}$ \\
\hline UNIQUAC & Aktivitätskoeffizientenmodell (Universal Quasichemical) \\
\hline LL & Flüssig-Flüssig (Liquid-Liquid) \\
\hline VLL & Dampf-Flüssig-Flüssig (Vapour-Liquid-Liquid) \\
\hline
\end{tabular}

\section{Lateinische Symbole}

\begin{tabular}{|c|c|c|}
\hline $\mathbf{A}$ & & stöchiometrische Matrix des Gesamtsystems \\
\hline $\mathbf{A}_{\mathrm{p}}$ & & stöchiometrische Matrix der Phasenübergänge \\
\hline $\mathbf{A}_{\mathrm{r}}^{\pi}, \mathbf{A}_{\mathrm{r}}$ & & $\begin{array}{l}\text { stöchiometrische Matrix der chemischen Reaktionen in } \\
\text { Phase } \pi \text { bzw. in allen Phasen }\end{array}$ \\
\hline$f_{\alpha}^{\pi}, f_{\alpha}^{0}$ & {$[\mathrm{~Pa}]$} & $\begin{array}{l}\text { Fugazität der Komponente } \alpha \text { in Phase } \pi \text {, bzw. Fugazität der } \\
\text { reinen Komponente }\end{array}$ \\
\hline G & {$[\mathrm{J}]$} & Gibbs-Energie \\
\hline $\begin{array}{l}\Delta_{\mathrm{r}} g_{\rho}^{\pi} \\
\Delta_{\mathrm{m}} g_{\alpha}^{\pi, \pi^{\prime}}\end{array}$ & {$\left[\mathrm{J} \mathrm{mol}^{-1}\right]$} & $\begin{array}{l}\text { Änderung der molaren Gibbs-Energie infolge Reaktion bzw. } \\
\text { Phasenübergang }\end{array}$ \\
\hline I & & Einheitsmatrix \\
\hline$k_{\alpha}^{\pi, \pi^{\prime}}, k_{\rho}^{\pi}$ & & beliebige positive Ratenkonstanten \\
\hline$K_{\mathrm{eq}, \rho}^{\pi}$ & & Gleichgewichtskonstante der Reaktion $\rho$ in Phase $\pi$ \\
\hline$n_{\alpha}^{\pi}$ & {$[\mathrm{mol}]$} & Stoffmenge der Komponente $\alpha$ in Phase $\pi$ \\
\hline $\mathbf{n}^{\pi}, \mathbf{n}$ & [mol] & Vektor der Stoffmengen in Phase $\pi$ bzw. im Gesamtsystem \\
\hline$P, P^{\theta}$ & {$[\mathrm{Pa}]$} & Druck bzw. Standarddruck \\
\hline $\mathcal{P}$ & & Menge aller im System auftretenden Phasen \\
\hline$r_{\alpha}^{\pi, \pi^{\prime}}$ & {$\left[\mathrm{mol} \mathrm{s}^{-1}\right]$} & $\begin{array}{l}\text { Rate des Stofftransports der Komponente } \alpha \text { zwischen den } \\
\text { Phasen } \pi \text { und } \pi^{\prime}\end{array}$ \\
\hline $\mathbf{r}^{\pi, \pi^{\prime}}, \mathbf{r}_{\mathrm{p}}$ & {$\left[\mathrm{mol} \mathrm{s}^{-1}\right]$} & $\begin{array}{l}\text { Vektor der Raten zwischen Phasen } \pi \text { und } \pi^{\prime} \text { bzw. aller } \\
\text { Raten für Phasenübergänge }\end{array}$ \\
\hline$r_{\rho}^{\pi}$ & {$\left[\mathrm{mol} \mathrm{s}^{-1}\right]$} & Rate infolge der chemischen Reaktion $\rho$ in Phase $\pi$ \\
\hline $\mathbf{r}^{\pi}, \mathbf{r}_{\mathrm{r}}$ & {$\left[\mathrm{mol} \mathrm{s}^{-1}\right]$} & $\begin{array}{l}\text { Vektor der Raten infolge chemischer Reaktionen in Phase } \pi \\
\text { bzw. in allen Phasen }\end{array}$ \\
\hline $\mathbf{r}$ & {$\left[\mathrm{mol} \mathrm{s}^{-1}\right]$} & Vektor aller Raten \\
\hline$R$ & {$\left[\mathrm{~J} \mathrm{~mol}^{-1} \mathrm{~K}^{-1}\right]$} & Universelle Gaskonstante \\
\hline $\mathcal{R}^{\pi}$ & & Menge aller chemischen Reaktionen in Phase $\pi$ \\
\hline $\mathcal{S}$ & & Menge aller Spezies im System \\
\hline$T$ & {$[\mathrm{~K}]$} & Temperatur \\
\hline$x_{\alpha}^{\pi}$ & - & Molenbruch der Komponente $\alpha$ in Phase $\pi$ \\
\hline $\mathbf{x}^{\pi}$ & - & Vektor der Molenbrüche in Phase $\pi$ \\
\hline
\end{tabular}




\section{Griechische Symbole}

\begin{tabular}{|l|l|l|}
\hline$\alpha$ & & Index der sich auf eine Komponente bezieht \\
\hline$\gamma_{\alpha}^{\pi}$ & - & Aktivitätskoeffizient der Komponente $\alpha$ in Phase $\pi$ \\
\hline$\mu_{\alpha}^{\pi}, \mu_{\alpha}^{I G, \theta}$ & {$\left[\mathrm{J} \mathrm{mol}^{-1}\right]$} & $\begin{array}{l}\text { chemisches Potential der Komponente } \alpha \text { in Phase } \pi \text { bzw. } \\
\text { chemisches Potential im idealen Gaszustand bei } P^{\theta}\end{array}$ \\
\hline$v_{\alpha \rho}^{\pi}$ & - & $\begin{array}{l}\text { Stöchiometrischer Koeffizient der Komponente } \alpha \text { in Reaktion } \\
\rho \text { in Phase } \pi\end{array}$ \\
\hline$\pi$ & & Index der Phase \\
\hline$\rho$ & {$\left[\mathrm{J} \mathrm{K}^{-1} \mathrm{~s}^{-1}\right]$} & Index der chemischen Reaktion \\
\hline$\dot{\sigma}_{\mathrm{S}}$ & {$[\mathrm{s}]$} & Entropieproduktionsdichte \\
\hline$\tau$ & - & Zeit \\
\hline$\varphi_{\alpha}^{\pi}$ & - & Fugazitätskoeffizient der Komponente $\alpha$ in Phase $\pi$ \\
\hline$\xi$ & Reaktionslaufzahl \\
\hline
\end{tabular}

\section{Literatur}

[1] S. M. Walas, Phase Equilibria in Chemical Engineering, Butterworth Publishers, 1985.

[2] J. Gmehling, B. Kolbe, M. Kleiber, J. Rarey, Chemical Thermodynamics for Process Simulation, Wiley-VCH, 2012.

[3] Y. Lwin, Int. J. Engng. Ed., 2000, 16 (4), 335 - 339.

[4] B. E. Poling, J. M. Prausnitz, J. P. O'Connell, The Properties of Gases and Liquids, McGraw-Hill, 2007.

[5] A. Lucia, L. Padmanabhan, S. Venkataraman, Comput. Chem. Eng., 2000, 24 (12), $2557-2569$.

[6] C. M. McDonald, C. A. Floudas, Ind. Eng. Chem. Res., 1995, 34 (5), 1674 - 1687.

[7] F. E. Pereira, G. Jackson, A. Galindo, C. S. Adjiman, Fluid Phase Equilibr., 2010, 299 (1), $1-23$.

[8] M. H. A. Piro, S. Simunovic, Comput. Mater. Sci., 2016, 118, 87 - 96.

[9] A. Zinser, K. Ye, L. Rihko-Struckmann, K. Sundmacher, Comput. Aided Chem. Eng., 2015, 37, $299-304$.

[10] A. Zinser, L. Rihko-Struckmann, K. Sundmacher, Comput. Chem. Eng., 2016, 89, 1 10.

[11] A. Zinser, L. Rihko-Struckmann, K. Sundmacher, Comput. Aided Chem. Eng., 2016, 38, $517-522$.

[12] R. G. Ketchum, Chem. Eng. Sci., 1979, 34 (3), 387 - 395. 
[13] H. D. Baehr, S. Kabelac, Thermodynamik, Springer, 2009.

[14] S. R. de Groot, P. Mazur, Non-equilibrium Thermodynamics, Dover Publications, 2013.

[15] P. Kondepudi, I. Prigogine, Modern Thermodynamics - From Heat Engines to Dissipative Structures, Wiley, 1998.

[16] G. Soave, Chem. Eng. Sci., 1972, 27 (6), 1197 - 1203.

[17] H. Cheung, R. S. Tanke, G. E. Torrence, in: Ullmann's Encyclopedia of Industrial Chemistry, Wiley-VCH, 2000.

[18] G. Venimadhavan, M. F. Malone, M. F. Doherty, Ind. Eng. Chem. Res., 1999, 38, 714 722.

[19] L. F. Shampine, M. W. Reichelt, SIAM J. Sci. Comput., 1997, 18, 1 - 22.

[20] M. J, D. Powell, in: Numerical Methods for Nonlinear Algebraic Equations, Ch. 7, 1970.

[21] J. J. Moré, D. C. Sorensen, SIAM J. Sci. Stat. Comp., 1983, 3, 553 - 572.

[22] D. Marquardt, SIAM J. Appl. Math., 1963, 11, 431 - 441. 


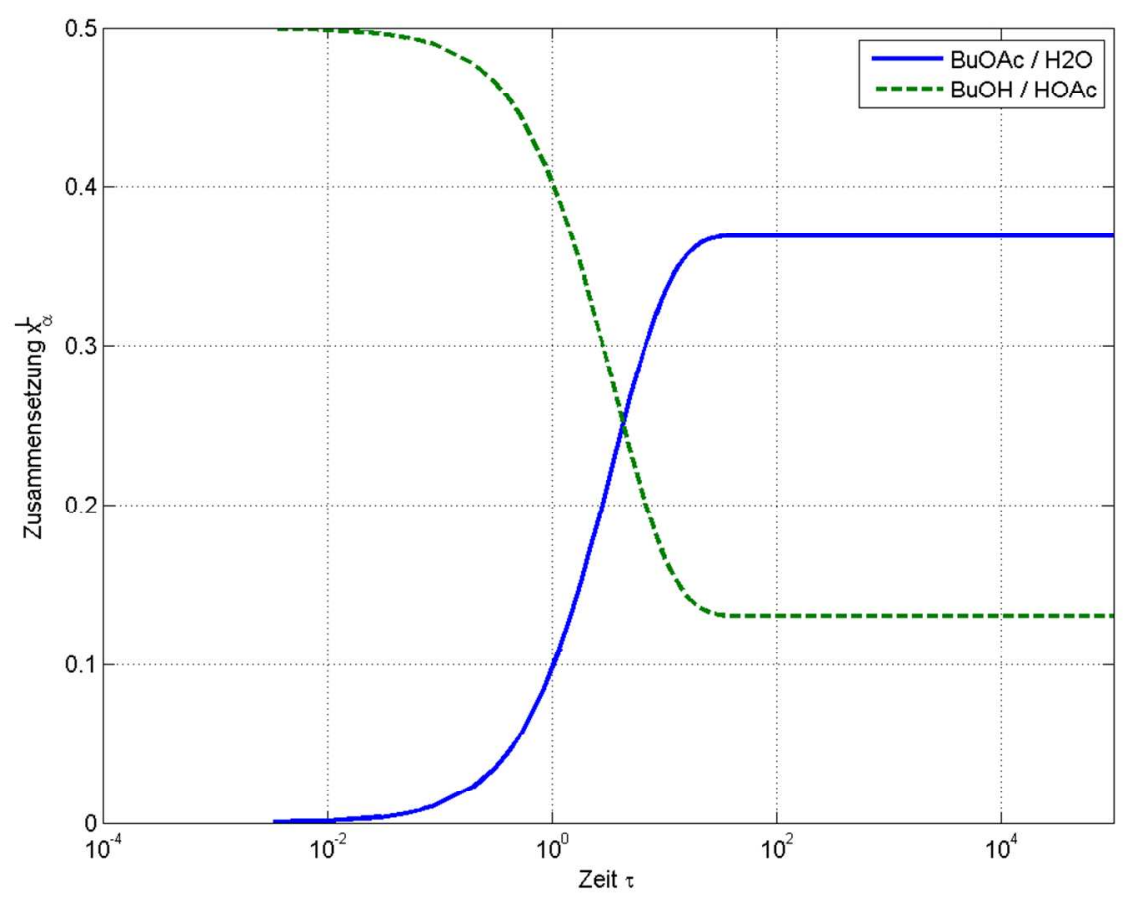

Abbildung 1: Entwicklung der Zusammensetzung infolge chemischer Reaktion. Abbildung 1 $203 \times 152 \mathrm{~mm}(150 \times 150 \mathrm{DPI})$ 
Abbildung 2: Entwicklung der Zusammensetzung in Dampf- und Flüssigphase über der Zeit. Abbildung 2 $203 \times 152 \mathrm{~mm}(150 \times 150 \mathrm{DPI})$ 
Abbildung 3: Trajektorien der Zusammensetzung für Reaktion und Separation im ternären Phasendiagramm. Abbildung 3 $203 \times 152 \mathrm{~mm}(150 \times 150$ DPI $)$ 

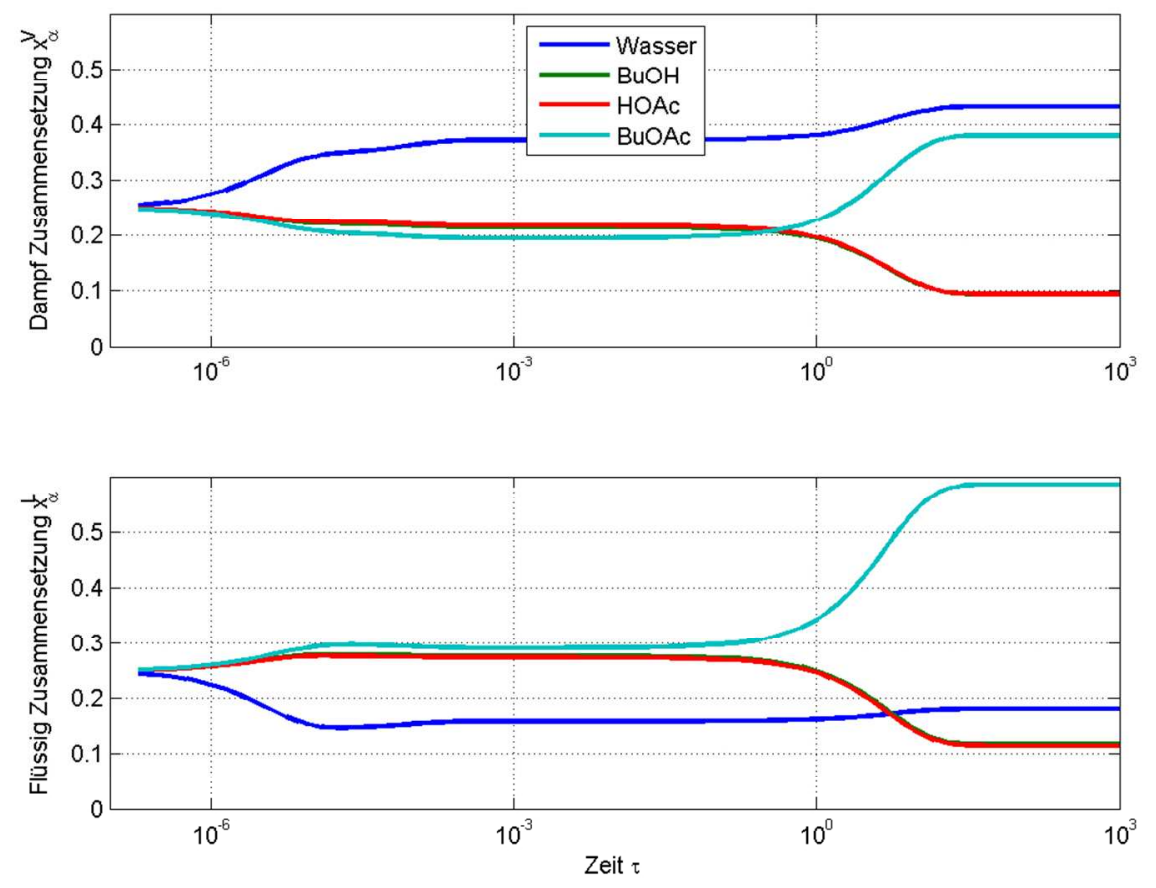

Abbildung 4: Entwicklung der Zusammensetzung in Dampf- und Flüssigphase über der Zeit im reaktiven System. Abbildung 4 $203 \times 152 \mathrm{~mm}(150 \times 150 \mathrm{DPI})$ 
Abbildung 5: Trajektorien der Zusammensetzung bei der simultanen Gleichgewichtsberechnung. Abbildung 5 $203 \times 152 \mathrm{~mm}(150 \times 150$ DPI $)$ 
Abbildung 6: Ternäres Phasendiagramm des Flüssig-Flüssig-Systems und Zweiphasengebiet. Abbildung 6 $203 \times 152 \mathrm{~mm}(150 \times 150$ DPI $)$ 

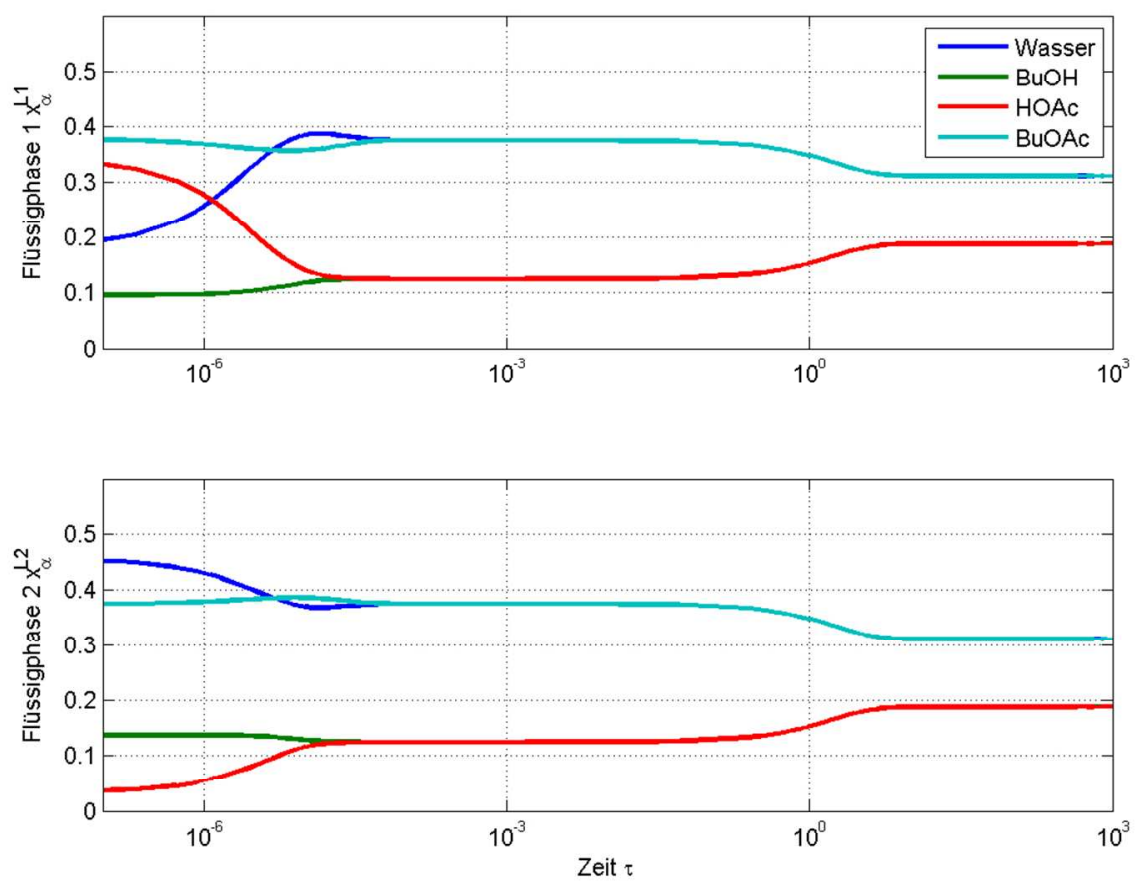

Abbildung 7: Entwicklung der Zusammensetzungen des reaktiven Flüssig-Flüssig-Systems über der Zeit. Abbildung 7 $203 \times 152 \mathrm{~mm}(150 \times 150 \mathrm{DPI})$ 